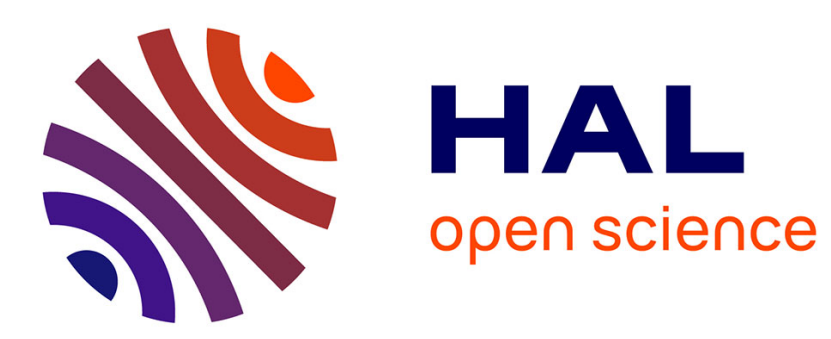

\title{
A Simple Method for Compressible Multiphase Mixtures and Interfaces
}

\author{
Nikolai Andrianov, Richard Saurel, Gerald Warnecke
}

\section{To cite this version:}

Nikolai Andrianov, Richard Saurel, Gerald Warnecke. A Simple Method for Compressible Multiphase Mixtures and Interfaces. RR-4247, INRIA. 2001. inria-00072340

\section{HAL Id: inria-00072340 https://hal.inria.fr/inria-00072340}

Submitted on 23 May 2006

HAL is a multi-disciplinary open access archive for the deposit and dissemination of scientific research documents, whether they are published or not. The documents may come from teaching and research institutions in France or abroad, or from public or private research centers.
L'archive ouverte pluridisciplinaire HAL, est destinée au dépôt et à la diffusion de documents scientifiques de niveau recherche, publiés ou non, émanant des établissements d'enseignement et de recherche français ou étrangers, des laboratoires publics ou privés. 


\section{A Simple Method for Compressible Multiphase Mixtures and Interfaces}

Nikolai Andrianov — Richard Saurel — Gerald Warnecke

\section{$\mathbf{N}^{\circ} \mathbf{4 2 4 7}$}

September 2001

THÈME 4

\section{apport}

derecherche 



\title{
A Simple Method for Compressible Multiphase Mixtures and Interfaces
}

\author{
Nikolai Andrianov, Richard Saure \\ Thème 4 - Simulation et optimisation \\ de systèmes complexes \\ Projet SMASH
}

Rapport de recherche $n^{\circ} 4247$ - September 2001 - 30 pages

\begin{abstract}
We develop a Godunov-type scheme for a non-conservative, unconditional hyperbolic multiphase model. It involves a set of seven partial differential equations and has the ability to solve interface problems between pure materials as well as compressible multiphase mixtures with two velocities and non-equilibrium thermodynamics (two pressures, two temperatures, two densities, etc.). Its numerical resolution poses several difficulties. The model possesses a large number of acoustic and convective waves (seven waves) and it is not easy to upwind all these waves accurately and simply. Also, the system is non-conservative, and the numerical approximations of the corresponding terms need to be provided. In this paper we focus on a method, based on a characteristic decomposition which solves these problems in a simple way and with good accuracy. The robustness, accuracy and versatility of the method is clearly demonstrated on several test problems with exact solutions.
\end{abstract}

Key-words: Two-phase flows, hyperbolic models, non-conservative equations, Godunov methods, interface problems.

* IAN, Otto-von-Guericke Universität Magdeburg, PSF 4120, D-39016 Magdeburg, Germany. Email: nikolai.andrianov@mathematik.uni-magdeburg.de

$\dagger$ Institut Universitaire des Systèmes Thermiques Industriels, 5 rue Enrico Fermi, 13453 Marseille Cedex 13, France and INRIA Projet SMASH, 2004 route des Lucioles, 06902 Sophia Antipolis. Email: richard@iusti.univ-mrs.fr

‡ IAN, Otto-von-Guericke Universität Magdeburg, PSF 4120, D-39016 Magdeburg, Germany. Email: gerald.warnecke@mathematik.uni-magdeburg.de 


\section{Une méthode simple pour le calcul des mélanges compressibles multiphasiques et des interfaces}

Résumé : Nous developpons un schéma de type Godunov pour un système hyperbolique non-conservatif modélisant des écoulements multiphasiques. Le modèle est composé d'un ensemble de sept équations aux dérivées partielles et il a la capacité de calculer des problèmes à interfaces entre des matériaux purs aussi bien que des problèmes de mélanges multiphasiques compressibles à deux vitesses et avec une thermodynamique en non-equilibre (deux pression, deux températures, deux masses volumiques, etc...). Son approximation numérique pose plusieurs difficultés. Le modèle possède un grand nombre d'ondes acoustiques et convectives (sept ondes) et il n'est pas aisé de décentrer toutes ces ondes d'une manière simple et précise. De plus, le modèle est non-conservatif et une approximation numérique des termes non-conservatifs doit être développée. Dans ce travail, nous nous concentrons sur une méthode basée sur une décomposition caractéristique qui résout ces problèmes de façon simple et avec une bonne précision. La robustesse, précision et généralité de la méthode est clairement démontrée sur plusieurs problèmes test dont la solution exact est connue.

Mots-clés : Ecoulements diphasiques, modèles hyperboliques, équations non-conservatives, Schéma de Godunov, Problème d'interface 


\section{Introduction}

Multiphase flows are involved in a huge number of fundamental and industrial applications. Multiphase mixtures may have several origins. Usually they are consequences of a physical mixing process of several fluids or materials. But under some circumstances, they may come from artificial smearing of contact discontinuities separating fluids of different physical and chemical properties.

We consider here the numerical resolution of a compressible multiphase flow model, first proposed by Baer and Nunziato 2 for detonation waves in granular explosives, and modified in Saurel and Abgrall [15] for the resolution of multiphase mixtures and interface problems between pure compressible materials.

In this last reference the goal was to use the same numerical method for the physical problems involving two-phase mixtures with two velocities, as well as interface problems with the single pressure and velocity. This aim was reached by using:

- An unconditional hyperbolic model for fluid mixtures, i.e. the system remains hyperbolic for all admissible states,

- An accurate method for flux computation as well as for the non-conservative terms,

- Pressure and velocity relaxation procedures.

This model and numerical solution procedures have been applied to several difficult physical problems like detonation waves in multiphase mixtures, multidimensional interfaces under shock interaction, underwater explosions for example, and cavitation in liquids [14.

However, this method has some drawbacks. The Riemann solver used in [15] and in [14] was much too dissipative. It involved only two waves instead of seven. Consequently not all the waves were upwinded, and the numerical solution was not accurate enough, in particular for convective waves. Here we propose a simple way to account for these waves in the solver.

In the literature several ways of solving the system from [2, [15], which take into account all waves, can be found. One of the common approaches is to neglect the non-conservative terms in the system of governing equations. This is done e.g. in Gonthier and Powers [7], where the authors give the following reasons to do so. First, they note that even without the non-conservative terms the system remains consistent with the second law of thermodynamics. Then, for the applications they consider, deflagration-to-detonation transition (DDT), these terms do not play a significant role in the process. Finally, they mention that the results without non-conservative terms fit well to the experimental data. The resulting system could be then expressed in divergence form and the Roe method is used for its solution.

Another method of solving the same type of model with neglected non-conservative terms is proposed in Fedkiw et. al. [4. The advection equation for the volume fraction is written in conservative form, and the characteristic decomposition method with the upwinding described as follows is used to solve the system. Namely, the seven left eigenvectors are used to project into the characteristic field, while only the first six entries of the seven right eigenvectors are used to project back out of these fields.

$\mathrm{RR} \mathrm{n}^{\circ} 4247$ 
However, as we show in Section 3, neglecting the non-conservative terms could lead to unphysical solutions, in particular when considering interface problems between pure materials.

The methodology which we follow in this work was introduced in Saurel and Abgrall [15]. In contrast to the methods mentioned above, we do not neglect non-conservative terms. This allows us to preserve the conditions of uniformity for pressure and velocity by construction, see Section 3.

The numerical method which we employ here for the upwinding of the convective and acoustic contributions of the flux is due to Gallouet and Masella [5. It is based on the local resolution of the linearized Riemann problem. The numerical flux is defined following the Godunov scheme, as the physical flux at the interface value of the solution of the Riemann problem. Then the non-conservative terms are discretized following the lines of Saurel and Abgrall [15]. This approach makes use of the fact that homogeneous states in pressure and velocity should be preserved. Thus, for conservative systems, the scheme is conservative and consistent without fulfilling Roe's condition on the linearized Jacobian matrix. We do not need to compute the Roe matrix in order to have a conservative method, which could be quite costly and cumbersome for big systems or when using complicated equations of state.

This feature is especially useful for typical problems, arising in multiphase flow modelling, where we often have to complete the seven equation model by extra equations. These could be the conservation equation for the number of particles per unit volume, which is needed for the calculation of the particle diameter, drag force, etc. We can also consider the micromacro coupling effects like bubble pulsation, particle rotation, pore collapse, etc., see [6], [12. Such type of system involves more equations, but remains hyperbolic and has the same structure.

The paper is organized as follows. In Section 2 we briefly review the mathematical model. Section 3 is devoted to the discussion on the necessity of considering the non-conservative terms. Sections 4-6 describe the numerical method, and Section 7 contains some numerical examples. 


\section{Presentation of the model}

Let us denote the gas and liquid phase with the subscripts $g$ and $l$, respectively, and the interface parameters with the subscript "int". Let $\alpha_{k}$ be the volume fractions, $\rho_{k}$ the material densities, $P_{k}$ the pressures, and $E_{k}=e_{k}+u_{k}^{2} / 2$ the specific total energies for $k=g, l$. The parameters $\lambda$ and $\mu$ determine the relaxation rates of velocities and pressures of the phases, see [15]. In [15], the following definitions are proposed for the interface pressure $P_{\text {int }}$ and velocity $V_{\text {int }}$,

$$
\begin{aligned}
P_{\text {int }} & =\alpha_{g} P_{g}+\alpha_{l} P_{l}, \\
V_{\text {int }} & =\left(\alpha_{g} \rho_{g} u_{g}+\alpha_{l} \rho_{l} u_{l}\right) /\left(\alpha_{g} \rho_{g}+\alpha_{l} \rho_{l}\right) .
\end{aligned}
$$

Other choices are possible according to the physical situation of interest. Then the governing equations for the one-dimensional compressible two-phase flow are [15]

$$
\begin{aligned}
\frac{\partial \alpha_{g}}{\partial t}+V_{\mathrm{int}} \frac{\partial \alpha_{g}}{\partial x}= & \mu\left(P_{g}-P_{l}\right) \\
\frac{\partial \alpha_{g} \rho_{g}}{\partial t}+\frac{\partial \alpha_{g} \rho_{g} u_{g}}{\partial x}= & 0 \\
\frac{\partial \alpha_{g} \rho_{g} u_{g}}{\partial t}+\frac{\partial \alpha_{g} \rho_{g} u_{g}^{2}+\alpha_{g} P_{g}}{\partial x}= & P_{\mathrm{int}} \frac{\partial \alpha_{g}}{\partial x}+\lambda\left(u_{l}-u_{g}\right) \\
\frac{\partial \alpha_{g} \rho_{g} E_{g}}{\partial t}+\frac{\partial \alpha_{g} u_{g}\left(\rho_{g} E_{g}+P_{g}\right)}{\partial x}= & P_{\mathrm{int}} V_{\mathrm{int}} \frac{\partial \alpha_{g}}{\partial x}+\lambda\left(u_{l}-u_{g}\right) V_{\mathrm{int}} \\
\frac{\partial \alpha_{l} \rho_{l}}{\partial t}+\frac{\partial \alpha_{l} \rho_{l} u_{l}}{\partial x}= & -\mu P_{\mathrm{int}}\left(P_{g}-P_{l}\right) \\
\frac{\partial \alpha_{l} \rho_{l} u_{l}}{\partial t}+\frac{\partial \alpha_{l} \rho_{l} u_{l}^{2}+\alpha_{l} P_{l}}{\partial x}= & -P_{\mathrm{int}} \frac{\partial \alpha_{g}}{\partial x}-\lambda\left(u_{l}-u_{g}\right) \\
\frac{\partial \alpha_{l} \rho_{l} E_{l}}{\partial t}+\frac{\partial \alpha_{l} u_{l}\left(\rho_{l} E_{l}+P_{l}\right)}{\partial x}= & -P_{\mathrm{int}} V_{\mathrm{int}} \frac{\partial \alpha_{g}}{\partial x}-\lambda\left(u_{l}-u_{g}\right) V_{\mathrm{int}} \\
& +\mu P_{\mathrm{int}}\left(P_{g}-P_{l}\right) .
\end{aligned}
$$

The equations (2) are closed by two equations of state (EOS), here we use the stiffened gas $E O S$, and the saturation constraint for the volume fractions,

$$
\begin{aligned}
P_{k} & =\left(\gamma_{k}-1\right) \rho_{k} e_{k}-\gamma_{k} \pi_{k}, \\
\alpha_{g}+\alpha_{l} & =1
\end{aligned}
$$


where $\gamma_{k}$ and $\pi_{k}$ are constants, specific for each phase. For gas we take $\gamma_{g}=1.4, \pi_{g}=0$, for liquid $\gamma_{l}=4.4, \pi_{l}=6 \cdot 10^{8} \mathrm{~Pa}$. Any other convex equation of state may be used for the thermodynamical closure of the model.

In order to investigate the mathematical structure of (2) it is convenient to rewrite it in primitive variables,

$$
\frac{\partial \mathbf{W}}{\partial t}+\mathbf{A} \frac{\partial \mathbf{W}}{\partial x}=\mathbf{S},
$$

where

$$
\mathbf{W}=\left(\alpha_{g}, \rho_{g}, u_{g}, P_{g}, \rho_{l}, u_{l}, P_{l}\right),
$$

the vector $\mathbf{S}$ contains the non-differential source terms, and the matrix $\mathbf{A}$ is given as,

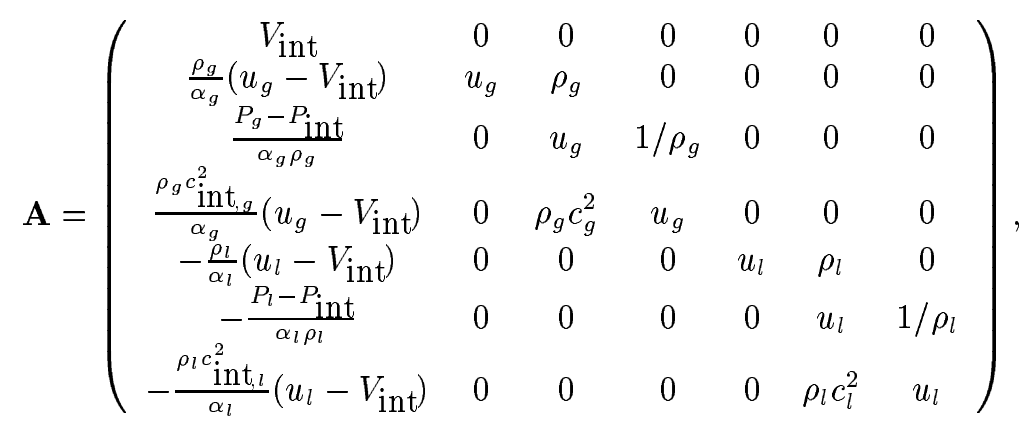

where

$$
c_{k}^{2}=\frac{\frac{p_{k}}{\rho_{k}^{2}}-\left.\frac{\partial e_{k}}{\partial \rho_{k}}\right|_{p_{k}}}{\left.\frac{\partial e_{k}}{\partial p_{k}}\right|_{\rho_{k}}} \quad \text { and } \quad c_{\mathrm{int}, k}^{2}=\frac{\frac{P_{\mathrm{int}}}{\rho_{k}^{2}}-\left.\frac{\partial e_{k}}{\partial \rho_{k}}\right|_{p_{k}}}{\left.\frac{\partial e_{k}}{\partial p_{k}}\right|_{\rho_{k}}}
$$

are the sound speeds for the phase $k$ and for the phase $k$ at the interface, respectively. A straightforward computation gives us the following expressions for the seven eigenvalues

$$
\begin{aligned}
& \lambda_{1}=V_{\text {int }}, \\
& \lambda_{2}=u_{g}+c_{g}, \quad \lambda_{3}=u_{g}-c_{g}, \quad \lambda_{4}=u_{g}, \\
& \lambda_{5}=u_{l}+c_{l}, \quad \lambda_{6}=u_{l}-c_{l}, \quad \lambda_{7}=u_{l} .
\end{aligned}
$$

The corresponding right eigenvectors are

$$
\mathbf{r}_{1}=\left[\begin{array}{l}
\alpha_{g} \alpha_{l} \sigma_{1} \sigma_{2} \\
-a_{l} \sigma_{2}\left(\rho_{g}\left(\sigma_{1}-c_{\mathrm{int}, g}^{2}\right)+P_{g}-P_{\mathrm{int}}\right), \\
\alpha_{l} \sigma_{2}\left(u_{g}-V_{\mathrm{int}}\right)\left(P_{g}-P_{\mathrm{int}}-\rho_{g} c_{\mathrm{int}, g}^{2}\right) / \rho_{g}, \\
a_{l} \sigma_{2}\left(\rho_{g} c_{\mathrm{int}, g}^{2}\left(u_{g}-V_{\mathrm{int}}\right)^{2}-c_{g}^{2}\left(P_{g}-P_{\mathrm{int}}\right)\right), \\
-a_{g} \sigma_{1}\left(\rho_{l}\left(c_{\mathrm{int}, l}^{2}-\sigma_{2}\right)-P_{l}+P_{\mathrm{int}}\right) \\
\alpha_{g} \sigma_{1}\left(u_{l}-V_{\mathrm{int}}\right)\left(-P_{l}+P_{\mathrm{int}}+\rho_{l} c_{\mathrm{int}, l}^{2}\right) / \rho_{l}, \\
a_{g} \sigma_{1}\left(-\rho_{l} c_{\mathrm{int}, l}^{2}\left(u_{l}-V_{\mathrm{int}}\right)^{2}+c_{l}^{2}\left(P_{l}-P_{\mathrm{int}}\right)\right)
\end{array}\right],
$$




$$
\begin{gathered}
\mathbf{r}_{2}=\left[\begin{array}{l}
0 \\
\rho_{g} \\
c_{g} \\
\rho_{g} c_{g}^{2} \\
0 \\
0 \\
0
\end{array}\right], \mathbf{r}_{3}=\left[\begin{array}{l}
0 \\
\rho_{g} \\
-c_{g} \\
\rho_{g} c_{g}^{2} \\
0 \\
0 \\
0
\end{array}\right], \mathbf{r}_{4}=\left[\begin{array}{l}
0 \\
1 \\
0 \\
0 \\
0 \\
0 \\
0
\end{array}\right], \\
\mathbf{r}_{5}=\left[\begin{array}{l}
0 \\
0 \\
0 \\
0 \\
\rho_{l} \\
c_{l} \\
\rho_{l} c_{l}^{2}
\end{array}\right], \mathbf{r}_{6}=\left[\begin{array}{l}
0 \\
0 \\
0 \\
0 \\
\rho_{l} \\
-c_{l} \\
\rho_{l} c_{l}^{2}
\end{array}\right], \mathbf{r}_{7}=\left[\begin{array}{l}
0 \\
0 \\
1 \\
0 \\
0
\end{array}\right],
\end{gathered}
$$

where

$$
\sigma_{1}=c_{g}^{2}-\left(u_{g}-V_{\mathrm{int}}\right)^{2}, \quad \sigma_{2}=c_{l}^{2}-\left(u_{l}-V_{\mathrm{int}}\right)^{2} .
$$

Therefore, the system (2) is hyperbolic, wherever the eigenvectors are linearly independent. Note that the system (2) is not strictly hyperbolic. Indeed, situations are possible, when some of the eigenvalues of the gas phase can coincide with some of the liquid phase. Moreover, it is easy to see that the eigenvectors (8)-(10) become linearly dependent in the points in the flow, where any one of conditions

$$
\alpha_{g}=0, \quad \alpha_{l}=0, \quad \sigma_{1}=0, \quad \text { or } \quad \sigma_{2}=0
$$

holds.

In this work we don't consider the mass and convective heat transfer terms, which have no importance for the design of the hyperbolic solver. The reader is referred to 14 for a description of such terms.

Note that the hyperbolicity of the model is only a consequence of the compressibility of the fluids. One can consider other assumptions on the interface parameters (1) without losing hyperbolicity.

$\mathrm{RR} \mathrm{n}^{\circ} 4247$ 


\section{Why are the non-conservative terms important}

We wish to show the importance of the non-conservative terms by using the following physical principle due to Abgrall [1]: a flow, uniform in velocity and pressure, must remain uniform during its temporal evolution. In other words, under the uniformity conditions on velocity and pressure, a contact discontinuity must be preserved.

Consider the mass and momentum conservation equations for one of the phases, we omit the subscript $g$ or $l$ for brevity,

$$
\begin{aligned}
\frac{\partial \alpha \rho}{\partial t}+\frac{\partial \alpha \rho u}{\partial x} & =0, \\
\frac{\partial \alpha \rho u}{\partial t}+\frac{\partial \alpha \rho u^{2}+\alpha P}{\partial x} & =\delta \cdot P_{\operatorname{int}} \frac{\partial \alpha}{\partial x} .
\end{aligned}
$$

Here the parameter $\delta=1$ for our model and $\delta=0$ for the models with neglected nonconservative terms [4], [7]. Let us show that with the second choice of $\delta$ the system does not preserve contact discontinuities.

Indeed, differentiating (11) out, we have

$$
\begin{aligned}
\frac{\partial \alpha \rho}{\partial t}+u \frac{\partial \alpha \rho}{\partial x}+\alpha \rho \frac{\partial u}{\partial x} & =0, \\
u \frac{\partial \alpha \rho}{\partial t}+\alpha \rho \frac{\partial u}{\partial t}+u^{2} \frac{\partial \alpha \rho}{\partial x}+\alpha \rho \frac{\partial u^{2}}{\partial x}+P \frac{\partial \alpha}{\partial x}+\alpha \frac{\partial P}{\partial x} & =\delta \cdot P_{\operatorname{int} \frac{\partial \alpha}{\partial x} .}
\end{aligned}
$$

Using the assumption of the uniform velocity and pressure field and the estimates (1), we get

$$
\begin{aligned}
P_{\text {int }}=P, \quad V_{\text {int }} & =u \\
\frac{\partial \alpha \rho}{\partial t}+u \frac{\partial \alpha \rho}{\partial x} & =0 \\
\frac{\partial P}{\partial x} & =0 \\
\frac{\partial u}{\partial x} & =0
\end{aligned}
$$

This implies that

$$
\alpha \rho \frac{\partial u}{\partial t}+P \frac{\partial \alpha}{\partial x}=\delta \cdot P \frac{\partial \alpha}{\partial x} .
$$

It appears clearly that in order for the flow to remain uniform with respect to velocity, i.e.

$$
\frac{\partial u}{\partial t}=0
$$

it is necessary that

$$
\delta=1
$$


The assumption $\delta=0$ is valid only in the particular and very restrictive case of

$$
\frac{\partial \alpha}{\partial x}=0
$$

Consider now the energy equation, to check that the pressure evolution is zero too,

$$
\frac{\partial \alpha \rho E}{\partial t}+\frac{\partial u(\alpha \rho E+\alpha P)}{\partial x}=\delta \cdot P_{\mathrm{int}} V_{\mathrm{int}} \frac{\partial \alpha}{\partial x} .
$$

Differentiating out and using the uniformity of velocity and pressure, we get

$$
\alpha \rho \frac{\partial E}{\partial t}+\alpha \rho u \frac{\partial E}{\partial x}+P u \frac{\partial \alpha}{\partial x}=\delta \cdot P u \frac{\partial \alpha}{\partial x} .
$$

By (14) one has

$$
\frac{\partial E}{\partial t}=\frac{\partial e}{\partial t},
$$

so (15) becomes

$$
\alpha \rho \frac{d e}{d t}+P u \frac{\partial \alpha}{\partial x}=\delta \cdot P u \frac{\partial \alpha}{\partial x},
$$

where $d / d t=\partial / \partial t+u \partial / \partial x$. Since $e=e(P, \rho)$,

$$
\frac{d e}{d t}=\left.\frac{\partial e}{\partial P}\right|_{\rho} \frac{d P}{d t}+\left.\frac{\partial e}{\partial \rho}\right|_{P} \frac{d \rho}{d t}
$$

and by (13),

$$
\frac{d e}{d t}=\left.\frac{\partial e}{\partial P}\right|_{\rho} \frac{\partial P}{\partial t}+\left.\frac{\partial e}{\partial \rho}\right|_{P} \frac{d \rho}{d t} .
$$

Using the advection equation for the volume fraction

$$
\frac{\partial \alpha}{\partial t}+u \frac{\partial \alpha}{\partial x}=0
$$

and the continuity equation (12) we get

$$
\frac{\partial \rho}{\partial t}+u \frac{\partial \rho}{\partial x}=0
$$

so that

$$
\frac{d \rho}{d t}=0
$$

hold. The energy equation becomes

$$
\left.\alpha \rho \frac{\partial e}{\partial P}\right|_{\rho} \frac{\partial P}{\partial t}+P u \frac{\partial \alpha}{\partial x}=\delta \cdot P u \frac{\partial \alpha}{\partial x},
$$

$\begin{array}{ll}\mathrm{RR} & \mathrm{n}^{\circ} 4247\end{array}$ 
which states again that

$$
\frac{\partial P}{\partial t}=0
$$

only if

$$
\delta=1 .
$$

Thus the flow remains uniform with respect to the velocity and pressure only when the non-conservative terms are considered.

Note that though in the case of a discontinuous flow the classical derivatives used in this section are not defined, we can get the preceding result in the sense of distributions. See Schwartz [16] for the general theory of distributions. Indeed, assuming the velocity and pressure fields to be uniform, we can rewrite the system (11) in a weak sense,

$$
\begin{gathered}
\left\langle\phi_{t}, \alpha \rho\right\rangle+u\left\langle\phi_{x}, \alpha \rho\right\rangle+\int_{-\infty}^{+\infty} \phi(x, 0) \alpha(x, 0) \rho(x, 0) d x=0 \\
u\left\langle\phi_{t}, \alpha \rho\right\rangle+u^{2}\left\langle\phi_{x}, \alpha \rho\right\rangle+\left\langle\phi_{x}, \alpha P\right\rangle- \\
\left\langle\phi_{x}, \delta P \alpha\right\rangle+u \int_{-\infty}^{+\infty} \phi(x, 0) \alpha(x, 0) \rho(x, 0) d x=0,
\end{gathered}
$$

where $\phi \in C_{0}^{1}(\mathbb{R} \times[0, \infty[)$ is a test function and

$$
\langle f, g\rangle=\int_{0}^{+\infty} \int_{-\infty}^{+\infty} f g d x d t .
$$

Multiplying (16) by $u$ and subtracting the result from (17), one gets

$$
\left\langle\phi_{x}, \alpha P\right\rangle=\left\langle\phi_{x}, \delta P \alpha\right\rangle .
$$

Using again the uniformity of pressure and the fact that the equality must hold for every $\phi$, one necessarily gets that

$$
\delta=1
$$




\section{Numerical method}

Following [15], we use the Strang splitting technique for the numerical solution of (2):

$$
\mathbf{V}_{i}^{n+1}=L_{s}^{\Delta t / 2} L_{h}^{\Delta t} L_{s}^{\Delta t / 2} \mathbf{V}_{i}^{n}
$$

where $\mathbf{V}_{i}^{n}$ is the vector of state variables on a mesh cell $i$ and time $t_{n}$, i.e.

$$
\mathbf{V}_{i}^{n}=\left(\alpha_{g}, \alpha_{g} \rho_{g}, \alpha_{g} \rho_{g} u_{g}, \alpha_{g} \rho_{g} E_{g}, \alpha_{l} \rho_{l}, \alpha_{l} \rho_{l} u_{l}, \alpha_{l} \rho_{l} E_{l}\right),
$$

$L_{h}$ is the operator of numerical solution of the hyperbolic part of the system (2), and $L_{s}$ is the operator of integration of the source and relaxation terms. Here we focus on the hyperbolic operator; the details on relaxation procedures may be found in Lallemand and Saurel [9].

\subsection{Hyperbolic operator}

The hyperbolic part of the system (2) can be rewritten in the following form,

$$
\begin{aligned}
& \frac{\partial \alpha_{g}}{\partial t}+V_{\text {int }} \frac{\partial \alpha_{g}}{\partial x}=0 \\
& \frac{\partial \mathbf{U}}{\partial t}+\frac{\partial \mathbf{f}(\mathbf{U})}{\partial x}=\mathbf{H} \frac{\partial \alpha_{g}}{\partial x},
\end{aligned}
$$

where $\mathbf{U}$ is given by

$$
\mathbf{U}_{i}=\left(\alpha_{g} \rho_{g}, \alpha_{g} \rho_{g} u_{g}, \alpha_{g} \rho_{g} E_{g}, \alpha_{l} \rho_{l}, \alpha_{l} \rho_{l} u_{l}, \alpha_{l} \rho_{l} E_{l}\right) .
$$

Following the idea of [15], we want to find a discretization of the non-conservative part of (2), i.e. the transport equation for $\alpha_{g}$ (18) and the term $\mathbf{H} \frac{\partial \alpha_{g}}{\partial x}$ in (19) in such a way, that the numerical approximation to the system would preserve a contact discontinuity. Let us write down the gas-related equations of (2), omitting the subscript $g$, the liquid part could be considered analogously,

$$
\begin{aligned}
\frac{\partial \alpha}{\partial t}+V_{\text {int } \frac{\partial \alpha}{\partial x}} & =0 \\
\frac{\partial \alpha \rho}{\partial t}+\frac{\partial \alpha \rho u}{\partial x} & =0 \\
\frac{\partial \alpha \rho u}{\partial t}+\frac{\partial \alpha \rho u^{2}+\alpha P}{\partial x} & =P_{\operatorname{int} \frac{\partial \alpha}{\partial x}} \\
\frac{\partial \alpha \rho E}{\partial t}+\frac{\partial \alpha u(\rho E+P)}{\partial x} & =P_{\text {int }} V_{\text {int }} \frac{\partial \alpha}{\partial x} .
\end{aligned}
$$

Let us denote by $\mathbf{f}$ is the physical flux function of (20), $\Delta$ some discrete form of $\frac{\partial \alpha}{\partial x}$, which is still to be determined, and $\mathbf{U}^{*}\left(\mathbf{U}_{j}^{n}, \mathbf{U}_{j+1}^{n}\right)$ the value of $\mathbf{U}$ along the line $x=x_{j+1 / 2}$ for the 


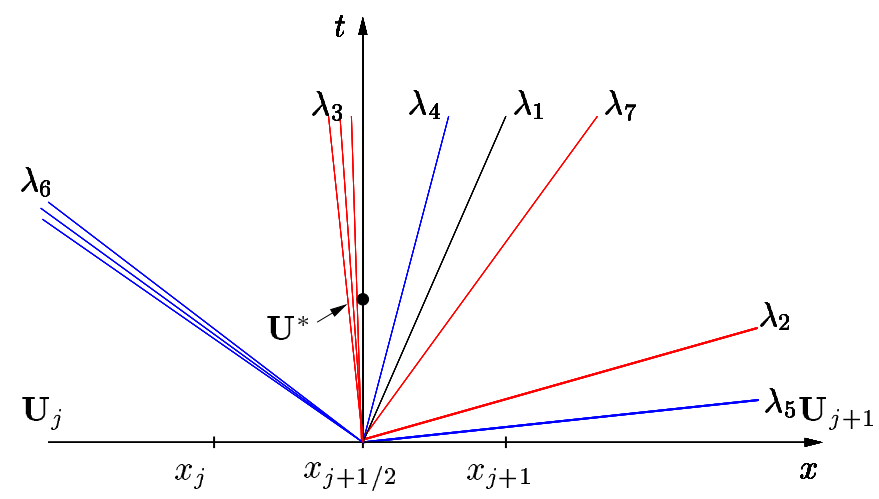

Figure 1: A typical Riemann problem, $\lambda_{i}$ are given by (7).

Riemann problem with the states $\mathbf{U}_{j}^{n}, \mathbf{U}_{j+1}^{n}$, see Fig. 1. Imagine we have some Godunov-type discretization of the last three equations, i.e.

$$
\mathbf{U}_{i}^{n+1}=\mathbf{U}_{i}^{n}-\frac{\Delta t}{\Delta x}\left[\mathbf{f}\left(\mathbf{U}^{*}\left(\mathbf{U}_{i}^{n}, \mathbf{U}_{i+1}^{n}\right)\right)-\mathbf{f}\left(\mathbf{U}^{*}\left(\mathbf{U}_{i-1}^{n}, \mathbf{U}_{i}^{n}\right)\right)\right]+\Delta t \mathbf{H} \Delta .
$$

Rewriting the scheme (21) componentwise, we get

$$
\begin{aligned}
(\alpha \rho)_{i}^{n+1}= & (\alpha \rho)_{i}^{n}-\frac{\Delta t}{\Delta x}\left[(\alpha \rho u)_{i+1 / 2}^{*}-(\alpha \rho u)_{i-1 / 2}^{*}\right] \\
(\alpha \rho u)_{i}^{n+1}= & (\alpha \rho u)_{i}^{n}-\frac{\Delta t}{\Delta x}\left[\left(\alpha \rho u^{2}+\alpha P\right)_{i+1 / 2}^{*}-\left(\alpha \rho u^{2}+\alpha P\right)_{i-1 / 2}^{*}\right] \\
& +\Delta t\left(P_{\mathrm{int}}\right)_{i}^{n} \Delta \\
(\alpha \rho E)_{i}^{n+1}= & (\alpha \rho E)_{i}^{n}-\frac{\Delta t}{\Delta x}\left[(\alpha \rho u E+\alpha P u)_{i+1 / 2}^{*}-(\alpha \rho u E+\alpha P u)_{i-1 / 2}^{*}\right] \\
& +\Delta t\left(P_{\mathrm{int}} V_{\mathrm{int}}\right)_{i}^{n} \Delta,
\end{aligned}
$$

where $*$ denotes the intermediate state.

Assume for the moment that the state $\mathbf{U}^{*}$ has been determined. Then discretizations for $\Delta$ and for the transport equation of $\alpha$ are obtained as follows. According to the principle due to Abgrall [1, a flow, uniform in pressure and velocity must remain uniform in the same variables during its time evolution. In other words if we had constant pressure and velocity everywhere in a flow at the time level $t_{n}$, then we will get the same pressure and velocity at the time $t_{n+1}$. Substituting constant pressures and velocities in the numerical scheme (22) we get

$$
\begin{aligned}
& u_{i}^{n}=u_{i}^{n+1}=u_{i \pm 1 / 2}^{*}=V_{\mathrm{int}}=u=\mathrm{const} \\
& P_{i}^{n}=P_{i}^{n+1}=P_{i \pm 1 / 2}^{*}=P_{\mathrm{int}}=P=\mathrm{const} .
\end{aligned}
$$


The first two equations of $[22]$ will be:

$$
\begin{aligned}
(\alpha \rho)_{i}^{n+1}= & (\alpha \rho)_{i}^{n}-u \frac{\Delta t}{\Delta x}\left[(\alpha \rho)_{i+1 / 2}^{*}-(\alpha \rho)_{i-1 / 2}^{*}\right] \\
(\alpha \rho)_{i}^{n+1} u= & (\alpha \rho)_{i}^{n} u-\frac{\Delta t}{\Delta x}\left[(\alpha \rho)_{i+1 / 2}^{*} u^{2}+\alpha_{i+1 / 2}^{*} P\right. \\
& \left.-(\alpha \rho)_{i-1 / 2}^{*} u^{2}-\alpha_{i-1 / 2}^{*} P\right]+\Delta t P \Delta .
\end{aligned}
$$

Multiplying (23) by $u$ and substracting the result from (24), we get the discretization for $\Delta$,

$$
\Delta=\frac{1}{\Delta x}\left(\alpha_{i+1 / 2}^{*}-\alpha_{i-1 / 2}^{*}\right) .
$$

Using the definition of $E$ and (25) in the last equation of (22), and combining it with (23), we get for internal energy,

$$
(\alpha \rho e)_{i}^{n+1}=(\alpha \rho e)_{i}^{n}-\frac{\Delta t}{\Delta x}\left[(\alpha \rho e)_{i+1 / 2}^{*} u-(\alpha \rho e)_{i-1 / 2}^{*} u\right] .
$$

Now using the equation of state (3) and the uniformity of pressure,

$$
\rho e=\frac{P+\gamma \pi}{\gamma-1}=\text { const }
$$

one gets

$$
\alpha_{i}^{n+1}=\alpha_{i}^{n}-u_{i}^{n} \frac{\Delta t}{\Delta x}\left(\alpha_{i+1 / 2}^{*}-\alpha_{i-1 / 2}^{*}\right),
$$

which is the discretized form of (18).

Thus, the Godunov-type scheme for the system (18)-(19) reads

$$
\begin{aligned}
\alpha_{i}^{n+1} & =\alpha_{i}^{n}-u_{i}^{n} \frac{\Delta t}{\Delta x}\left(\alpha_{i+1 / 2}^{*}-\alpha_{i-1 / 2}^{*}\right) \\
\mathbf{U}_{i}^{n+1} & =\mathbf{U}_{i}^{n}-\frac{\Delta t}{\Delta x}\left[\mathbf{f}\left(\mathbf{U}^{*}\left(\mathbf{U}_{i}^{n}, \mathbf{U}_{i+1}^{n}\right)\right)-\mathbf{f}\left(\mathbf{U}^{*}\left(\mathbf{U}_{i-1}^{n}, \mathbf{U}_{i}^{n}\right)\right)\right]+\Delta t \mathbf{H} \Delta,
\end{aligned}
$$

where $\Delta=\frac{1}{\Delta x}\left(\alpha_{i+1 / 2}^{*}-\alpha_{i-1 / 2}^{*}\right)$. In the following section we will specify $\mathbf{U}^{*}$, the intermediate value of the solution of the Riemann problem. 


\section{Approximate solution to the Riemann problem}

The numerical scheme (26) requires the solution of the Riemann problem at every cell boundary at each time step. In general, the exact solution of the system (2) is unknown. The determination of the Rankine-Hugoniot conditions and Riemann invariants is still an issue. Even if their expressions were known, the exact solution would be too complicated for computational purposes, so we have to use some approximate Riemann solver. The approach which we follow here is that due to Gallouet and Masella [5]. In this section we give the description of the original method, and its use in the case of the system (2).

\subsection{Conservative systems}

Consider a strictly hyperbolic system of $m$ conservation laws

$$
\mathbf{q}_{t}+\mathbf{g}(\mathbf{q})_{x}=0, \quad \mathbf{q}=\left(q_{1}, \ldots, q_{m}\right)^{T}
$$

with the initial data

$$
\mathbf{q}(x, 0)= \begin{cases}\mathbf{q}_{l}, & x \leq 0 \\ \mathbf{q}_{r}, & x>0\end{cases}
$$

Linearizing (27), one gets

$$
\mathbf{q}_{t}+\mathbf{B}\left(\overline{\mathbf{q}}\left(\mathbf{q}_{l}, \mathbf{q}_{r}\right)\right) \mathbf{q}_{x}=0
$$

The state $\overline{\mathbf{q}}$ is chosen in such a way, that $\mathbf{B}$ has real eigenvalues, e.g.

$$
\overline{\mathbf{q}}=\frac{\mathbf{q}_{l}+\mathbf{q}_{r}}{2} .
$$

For the linear problem (29), the solution of the Riemann problem can be found exactly, see LeVeque [10] for details. We introduce the characteristic variables

$$
\mathbf{s}=\mathbf{R}^{-1} \mathbf{q}, \quad \mathbf{s}=\left(s_{1}, \ldots, s_{m}\right)^{T},
$$

where $\mathbf{R}$ is the matrix of the right eigenvectors of $\mathbf{B}$. Then the system (27) decouples into $m$ scalar advection equations,

$$
s_{i t}+\lambda_{i} s_{i x}=0, \quad i=1, \ldots, m,
$$

where $\lambda_{i}$ are the eigenvalues of $\mathbf{B}$. The initial data (28) in characteristic variables will be

$$
\mathbf{s}(x, 0)=\left\{\begin{aligned}
\mathbf{s}_{l}=\mathbf{R}^{-1} \mathbf{q}_{l}=\left(\begin{array}{l}
s_{l 1} \\
\vdots \\
s_{l m}
\end{array}\right), & x \leq 0 \\
\mathbf{s}_{r}=\mathbf{R}^{-1} \mathbf{q}_{r}=\left(\begin{array}{l}
s_{r 1} \\
\vdots \\
s_{r m}
\end{array}\right), & x>0 .
\end{aligned}\right.
$$


Thus one has

$$
\begin{aligned}
\mathbf{q}_{r}-\mathbf{q}_{l} & =\mathbf{R}\left(\mathbf{s}_{r}-\mathbf{s}_{l}\right)=\mathbf{R}\left(\begin{array}{c}
s_{r 1}-s_{l 1} \\
\vdots \\
s_{r m}-s_{l m}
\end{array}\right) \\
& =\left(s_{r 1}-s_{l 1}\right) \mathbf{r}_{1}+\ldots+\left(s_{r m}-s_{l m}\right) \mathbf{r}_{m}=\sum_{i=1}^{m} b_{i} \mathbf{r}_{i}
\end{aligned}
$$

where $b_{i}=s_{r i}-s_{l i}$ and $\mathbf{r}_{i}$ are the right eigenvectors of $\mathbf{B}$. The intermediate state in the solution of Riemann problem is given by

$$
\mathbf{q}^{*}=\mathbf{q}_{l}+\sum_{\lambda_{i}<0} b_{i} \mathbf{r}_{i} .
$$

Then the Godunov-type scheme for the conservation law (27) reads

$$
\mathbf{q}_{i}^{n+1}=\mathbf{q}_{i}^{n}-\frac{\Delta t}{\Delta x}\left[\mathbf{g}\left(\mathbf{q}^{*}\left(\mathbf{q}_{i}^{n}, \mathbf{q}_{i+1}^{n}\right)\right)-\mathbf{g}\left(\mathbf{q}^{*}\left(\mathbf{q}_{i-1}^{n}, \mathbf{q}_{i}^{n}\right)\right)\right] .
$$

The scheme is conservative and has a consistent numerical flux. Provided we use entropysatisfying Riemann solutions $\mathbf{q}^{*}$, the weak solutions obtained by this scheme satisfy the entropy condition.

It is well known that the approximate solution of the Riemann problem, given by (30) could be non-physical (rarefaction shocks). To avoid this, the approach of Harten and Hyman [8] is used.

\subsection{Non-conservative systems}

Consider the Riemann problem for the hyperbolic part of the system (2), written in the primitive variable formulation (44)-(6),

$$
\frac{\partial \mathbf{W}}{\partial t}+\mathbf{A} \frac{\partial \mathbf{W}}{\partial x}=0 .
$$

The initial conditions are

$$
\mathbf{W}(x, 0)= \begin{cases}\mathbf{W}_{l}, & x \leq 0 \\ \mathbf{W}_{r}, & x>0 .\end{cases}
$$

Following [5], we calculate the Jacobian matrix $\mathbf{A}(\overline{\mathbf{W}})$ in the average state

$$
\overline{\mathbf{W}}=\frac{\mathbf{W}_{l}+\mathbf{W}_{r}}{2} .
$$

The intermediate state in the solution of the Riemann problem (33)-(32) is

$$
\mathbf{W}^{*}=\mathbf{W}_{l}+\sum_{\lambda_{i}<0} a_{i} \mathbf{r}_{i}
$$

$\mathrm{RR} \mathrm{n}^{\circ} 4247$ 
where the eigenvalues $\lambda_{i}$ and the corresponding eigenvectors $\mathbf{r}_{i}$ of the matrix $\mathbf{A}(\overline{\mathbf{W}})$ are given by (17), (8)-(10), and $a_{i}$ are the coefficients of eigenvector decomposition of $\mathbf{W}_{r}-\mathbf{W}_{l}$,

$$
\mathbf{W}_{r}-\mathbf{W}_{l}=\sum_{\lambda_{i}} a_{i} \mathbf{r}_{i} .
$$

For the system (2) they are given by the following expressions,

$$
\begin{aligned}
& a_{1}=\delta_{1} / r_{11}, \\
& a_{2}=\frac{\delta_{3} \rho_{g} c_{g}+\delta_{4}-a_{1}\left(r_{13} \rho_{g} c_{g}+r_{14}\right)}{2 \rho_{g} c_{g}^{2}}, \\
& a_{3}=\frac{-\delta_{3} \rho_{g} c_{g}+\delta_{4}+a_{1}\left(r_{13} \rho_{g} c_{g}-r_{14}\right)}{2 \rho_{g} c_{g}^{2}} \\
& a_{4}=\delta_{2}-a_{1} r_{12}-\rho_{g}\left(a_{2}+a_{3}\right), \\
& a_{5}=\frac{\delta_{6} \rho_{l} c_{l}+\delta_{7}-a_{1}\left(r_{16} \rho_{l} c_{l}+r_{17}\right)}{2 \rho_{l} c_{l}^{2}}, \\
& a_{6}=\frac{-\delta_{6} \rho_{l} c_{l}+\delta_{7}+a_{1}\left(r_{16} \rho_{l} c_{l}-r_{17}\right)}{2 \rho_{l} c_{l}^{2}} \\
& a_{7}=\delta_{5}-a_{1} r_{15}-\rho_{l}\left(a_{5}+a_{6}\right),
\end{aligned}
$$

where $r_{1 k}$ are the components of $\mathbf{r}_{1}, \delta_{k}$ is the $k$-th component of $\mathbf{W}_{r}-\mathbf{W}_{l}$.

Recalculating $\mathbf{W}^{*}$ into the conservative vector $\mathbf{U}^{*}$, we fully determine the Godunov-type scheme (26) for the system (2). 


\section{Extension to the second order}

We use the MUSCL approach to achieve the second order, which consists of three steps, namely

- Extrapolation. Given piecewise-constant values $\mathbf{W}_{i}^{n}$, we obtain the linearly extrapolated values

$$
\mathbf{W}_{i-1 / 2}^{+}=\mathbf{W}_{i}^{n}-\frac{1}{2} \bar{\sigma}_{i}, \quad \mathbf{W}_{i+1 / 2}^{-}=\mathbf{W}_{i}^{n}+\frac{1}{2} \bar{\sigma}_{i} .
$$

The essential issue is that this step is performed in primitive variables; this ensures preservation of uniformity of pressure and velocity. The limited slopes $\bar{\sigma}_{i}$ are taken equal to

$$
\bar{\sigma}_{i}= \begin{cases}\max \left[0, \min \left(\beta \Delta_{i-1 / 2}, \Delta_{i+1 / 2}\right), \min \left(\Delta_{i-1 / 2}, \beta \Delta_{i+1 / 2}\right)\right], & \Delta_{i+1 / 2}>0 \\ \min \left[0, \max \left(\beta \Delta_{i-1 / 2}, \Delta_{i+1 / 2}\right), \max \left(\Delta_{i-1 / 2}, \beta \Delta_{i+1 / 2}\right)\right], & \Delta_{i+1 / 2}<0\end{cases}
$$

where

$$
\Delta_{i-1 / 2}=\mathbf{W}_{i}^{n}-\mathbf{W}_{i-1}^{n}, \quad \Delta_{i+1 / 2}=\mathbf{W}_{i+1}^{n}-\mathbf{W}_{i}^{n} .
$$

In particular, $\beta=1$ corresponds to the minmod limiter, $\beta=2$ to the superbee limiter.

- Evolution. We evolve the values of $\mathbf{W}_{i \mp 1 / 2}^{ \pm}$according to

$$
\begin{aligned}
& \overline{\mathbf{W}}_{i-1 / 2}^{+}=\mathbf{W}_{i-1 / 2}^{+}-\frac{\Delta t}{2 \Delta x} \mathbf{A}\left(\mathbf{W}_{i}\right)\left(\mathbf{W}_{i+1 / 2}^{-}-\mathbf{W}_{i-1 / 2}^{+}\right) \\
& \overline{\mathbf{W}}_{i+1 / 2}^{-}=\mathbf{W}_{i+1 / 2}^{-}-\frac{\Delta t}{2 \Delta x} \mathbf{A}\left(\mathbf{W}_{i}\right)\left(\mathbf{W}_{i+1 / 2}^{-}-\mathbf{W}_{i-1 / 2}^{+}\right)
\end{aligned}
$$

- The Riemann problem. We rewrite the vectors $\overline{\mathbf{W}}_{j \pm 1 / 2}^{ \pm}$in conservative variables, solve the Riemann problems with piecewise constant data $\left(\overline{\mathbf{U}}_{j+1 / 2}^{-}, \overline{\mathbf{U}}_{j+1 / 2}^{+}\right)$and get the intermediate states $\mathbf{U}_{j+1 / 2}^{*}$.

Then the numerical scheme (second order in space and time) reads

$$
\begin{aligned}
\alpha_{i}^{n+1}= & \alpha_{i}^{n}-V_{\mathrm{int}}^{*} \frac{\Delta t}{\Delta x}\left(\bar{\alpha}_{i+1 / 2}^{*}-\bar{\alpha}_{i-1 / 2}^{*}\right) \\
\mathbf{U}_{i}^{n+1}= & \mathbf{U}_{i}-\frac{\Delta t}{\Delta x}\left[\mathbf{f}\left(\mathbf{U}^{*}\left(\overline{\mathbf{U}}_{i+1 / 2}^{-}, \overline{\mathbf{U}}_{i+1 / 2}^{+}\right)\right)-\mathbf{f}\left(\mathbf{U}^{*}\left(\overline{\mathbf{U}}_{i-1 / 2}^{-}, \overline{\mathbf{U}}_{i-1 / 2}^{+}\right)\right)\right] \\
& +\Delta t \mathbf{H} \Delta,
\end{aligned}
$$

where $\Delta=\frac{1}{\Delta x}\left(\bar{\alpha}_{i+1 / 2}^{*}-\bar{\alpha}_{i-1 / 2}^{*}\right)$. 


\section{$7 \quad$ Numerical examples}

To illustrate the properties of the new scheme we have chosen essentially the same test problems as in reference [15]. Each problem was solved with the new scheme and with the original method of the reference [15]. In what follows, the new scheme will be referred to as VFRoe, stands for Volumes Finis Roe in French, notion taken from Gallouet and Masella [5]. The original method of the reference [15] will be referred to as HLL due to the Harten-Lax-van Leer numerical flux function used there. The calculations were made with the second order accurate scheme using a CFL number of 0.9. The numerical results were then compared with the exact solution for the first two problems and with the experimental data for the third problem.

\subsection{Water-air shock tube}

We consider the shock tube filled with liquid water under high pressure at the left, and with the gas(air) at the right. Each fluid is governed by the stiffened gas EOS

$$
P_{k}=\left(\gamma_{k}-1\right) \rho_{k} e_{k}-\gamma_{k} \pi_{k}, \quad k=g, l,
$$

with the following parameters

$$
\begin{array}{ll}
\text { Liquid } & \text { Gas } \\
\gamma_{l}=4.4 & \gamma_{g}=1.4 \\
\pi_{l}=6 \cdot 10^{8} & \pi_{g}=0 .
\end{array}
$$

The initial conditions for the system (2) are

$$
\begin{aligned}
& \text { Left: } \quad x \leq 0.7 \\
& \rho_{l}=1000 \mathrm{~kg} / \mathrm{m}^{3} \\
& P_{l}=10^{9} \mathrm{~Pa} \\
& \text { Right: } \quad x>0.7 \\
& \rho_{g}=50 \mathrm{~kg} / \mathrm{m}^{3} \\
& u_{l}=0 \mathrm{~m} / \mathrm{s} \\
& P_{g}=10^{6} \mathrm{~Pa} \\
& \alpha_{l}=1 \\
& u_{g}=0 \mathrm{~m} / \mathrm{s}
\end{aligned}
$$

Note that at the both sides of the interface the system (2) reduces to the Euler equations for liquid and gas. The solution of the Riemann problem (2)-(34) is schematically depicted in Fig. 2 Using that

$$
P_{g}^{*}=P_{l}^{*}, \quad u_{g}^{*}=u_{l}^{*},
$$

we can get the exact solution to the Riemann problem.

To solve it numerically, we allow the presence of a negligible small amount of gas, e.g. $\alpha_{g}=10^{-8}$, at the left of the shock tube, and a small amount of water at the right. Thus 


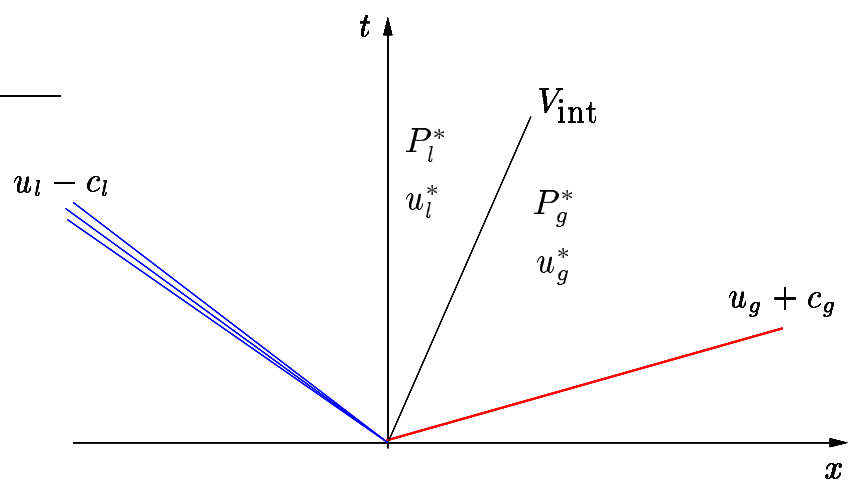

Figure 2: The Riemann problem (2)-(34). The interface $x / t=V_{\text {int }}$ separates the phases, liquid at the left and gas at the right.

the initial data will be as follows,

$$
\begin{aligned}
& \text { Left: } \quad x \leq 0.7 \\
& \rho_{g}=50 \mathrm{~kg} / \mathrm{m}^{3} \\
& \rho_{l}=1000 \mathrm{~kg} / \mathrm{m}^{3} \\
& P_{g}=P_{l}=10^{9} \mathrm{~Pa} \\
& \text { Right: } \quad x>0.7 \\
& \rho_{g}=50 \mathrm{~kg} / \mathrm{m}^{3} \\
& \rho_{l}=1000 \mathrm{~kg} / \mathrm{m}^{3} \\
& P_{g}=P_{l}=10^{6} \mathrm{~Pa} \\
& u_{g}=u_{l}=0 \mathrm{~m} / \mathrm{s} \\
& u_{g}=u_{l}=0 \mathrm{~m} / \mathrm{s} \\
& \alpha_{g}=10^{-8} \\
& \alpha_{l}=10^{-8} \text {. }
\end{aligned}
$$

For this problem, we use both the pressure and velocity relaxation procedures. We consider the exact solution of (2)-(34) to be a reference solution for the numerical solution of (2)-(35), having in mind that we have the exact solution for the liquid at the left, and for the gas at the right. 

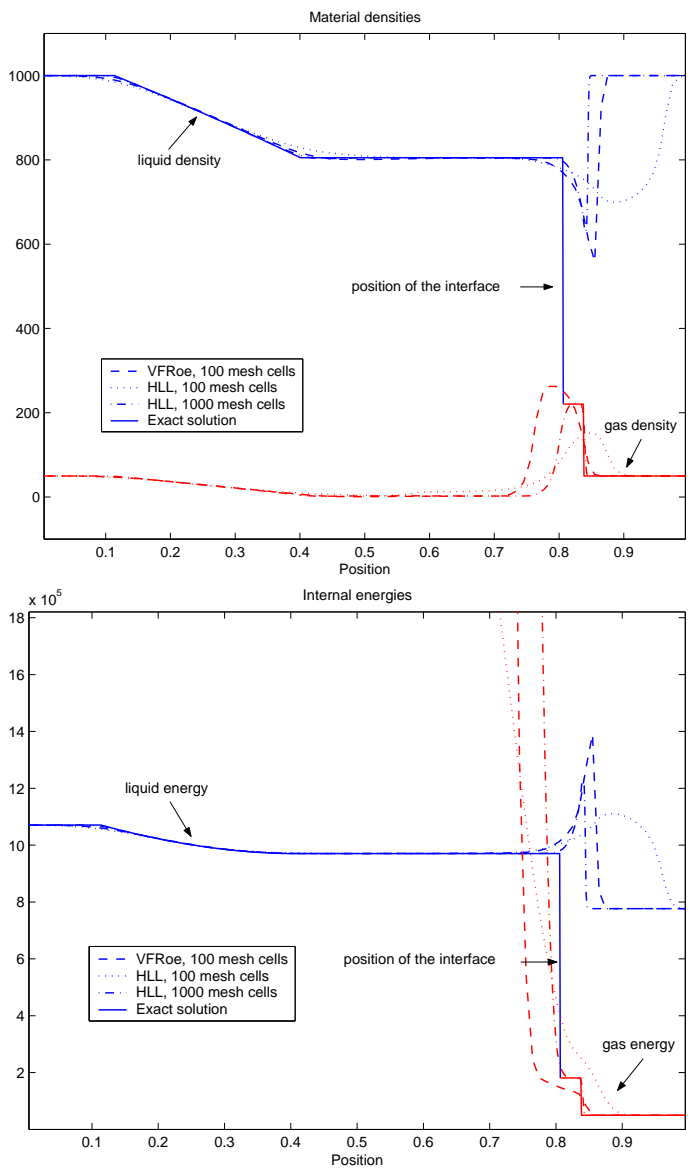

Figure 3: Water-air shock tube, material densities and energies of the phases.

The comparison of the numerical results for the VFRoe and HLL solvers with the exact solution at time $t=2.2 \mathrm{e}-4$ is presented in Fig. 3.5. As expected, the VFRoe solver gives a much sharper resolution of discontinuities compared to HLL. The numerical results, obtained with the VFRoe scheme over 100 mesh cells are comparable to the results of HLL over 300 mesh cells. The calculations with 1000 mesh cells show that the shock speeds are also correctly computed.

In Fig. 31, some peaks are visible in the gas parameters at the left of the interface, and in the liquid parameters to the right of it. We have observed this behavior of the solution with the both HLL and VFRoe schemes. As mentioned before, it is not possible to fully compare 

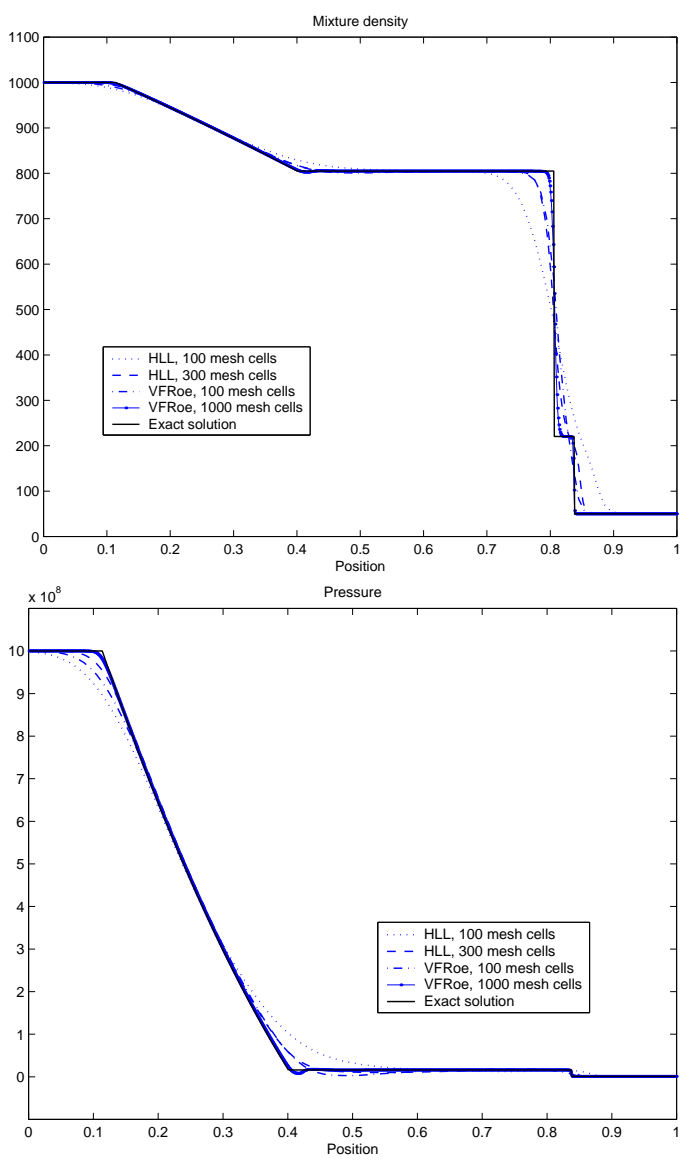

Figure 4: Water-air shock tube, mixture density and pressure.

these numerical results for initial data (35) with the reference solution to the system (2) with initial data (34).

In Fig. 45 the distributions of the mixture density,

$$
\rho_{\text {mix }}=\alpha_{g} \rho_{g}+\alpha_{l} \rho_{l},
$$

the relaxed pressures and velocities

$$
P=P_{g}=P_{l}
$$

$\mathrm{RR} \mathrm{n}^{\circ} 4247$ 

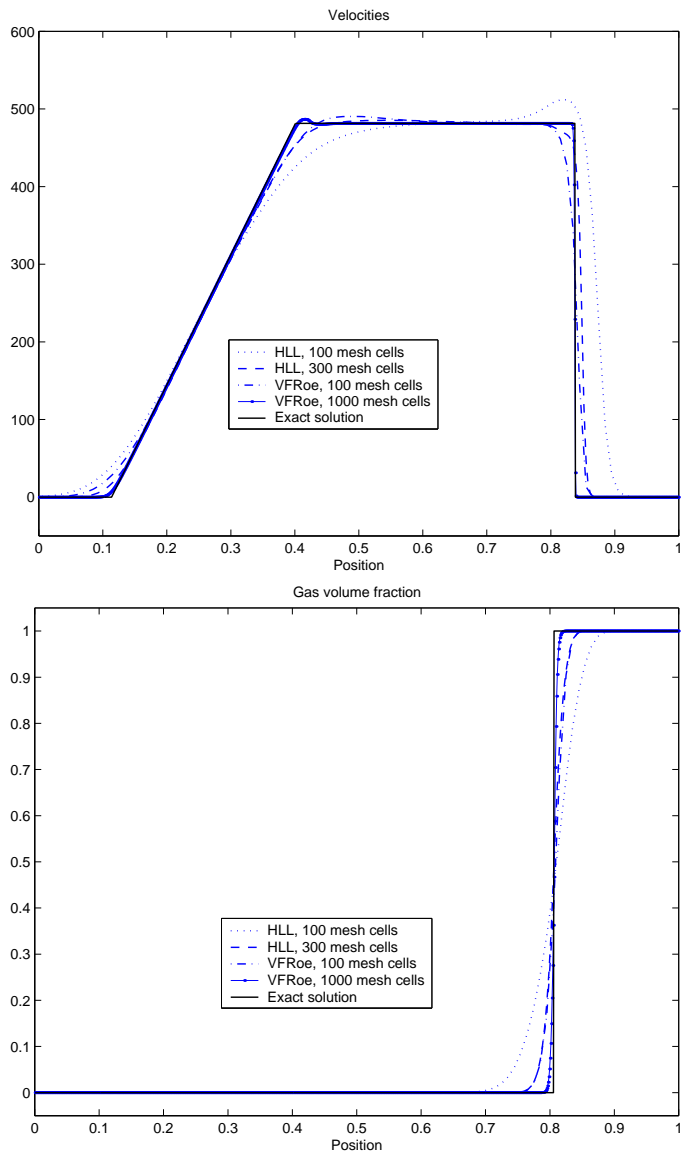

Figure 5: Water-air shock tube, velocities and gas volume fraction.

$$
u=u_{g}=u_{l},
$$

and gas volume fraction $\alpha_{g}$ are presented. The reference solution for the gas volume fraction is obtained as follows. Knowing the interface velocity $V_{\text {int }}$ from the solution of the Riemann problem (2) with initial data (34), we find the displacement of the interface $\Delta S$ over the time $\Delta t$ as

$$
\Delta S=V_{\text {int }} \Delta t
$$

In some extreme situations with very large ratios of initial pressures and densities, the VFRoe solver fails to preserve positivity of density of pressure in the intermediate state of the Riemann problem. The problem of positivity preservation of the VFRoe solver is an interesting question and will be the subject of future research. 

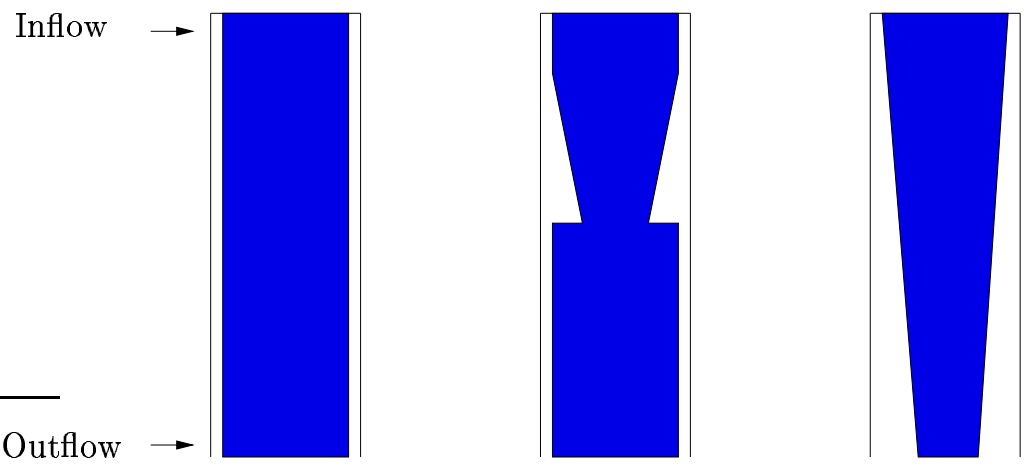

Initial conditions Intermediate state Steady state

Figure 6: Water faucet problem.

\subsection{Water faucet problem}

This test, which is due to Ransom [13], consists of a vertical tube $12 \mathrm{~m}$ in length, which contains a liquid (water) column, surrounded by gas (air). At the top, the volume fractions and the liquid velocity are given, and the gas velocity is zero. The bottom is open to atmospheric conditions. Under the action of gravity, a narrowing of the liquid jet takes place. Several stages of the process are depicted in Fig. [6]

The initial conditions are as follows,

$$
\begin{array}{ll}
\text { Liquid } & \text { Gas } \\
\rho_{l}=1000 \mathrm{~kg} / \mathrm{m}^{3} & \rho_{g}=1 \mathrm{~kg} / \mathrm{m}^{3} \\
P_{l}=10^{5} \mathrm{~Pa} & P_{g}=10^{5} \mathrm{~Pa} \\
u_{l}=10 \mathrm{~m} / \mathrm{s} & u_{g}=0 \mathrm{~m} / \mathrm{s} \\
\alpha_{l}=0.8 & \alpha_{g}=0.2 .
\end{array}
$$

The boundary conditions are

$$
\begin{array}{lll} 
& \text { Liquid } & \text { Gas } \\
\text { Top(inflow) } & u_{l}=10 \mathrm{~m} / \mathrm{s} & u_{g}=0 \mathrm{~m} / \mathrm{s} \\
& \alpha_{l}=0.8 & \alpha_{g}=0.2 \\
\text { Bottom(outflow) } & P_{l}=10^{5} \mathrm{~Pa} & P_{g}=10^{5} \mathrm{~Pa} \\
& \alpha_{l}, \alpha_{g} \text { are extrapolated, }
\end{array}
$$

other flow variables are found by the solution of the boundary Riemann problem for the phases at the top and at the bottom of the tube. 


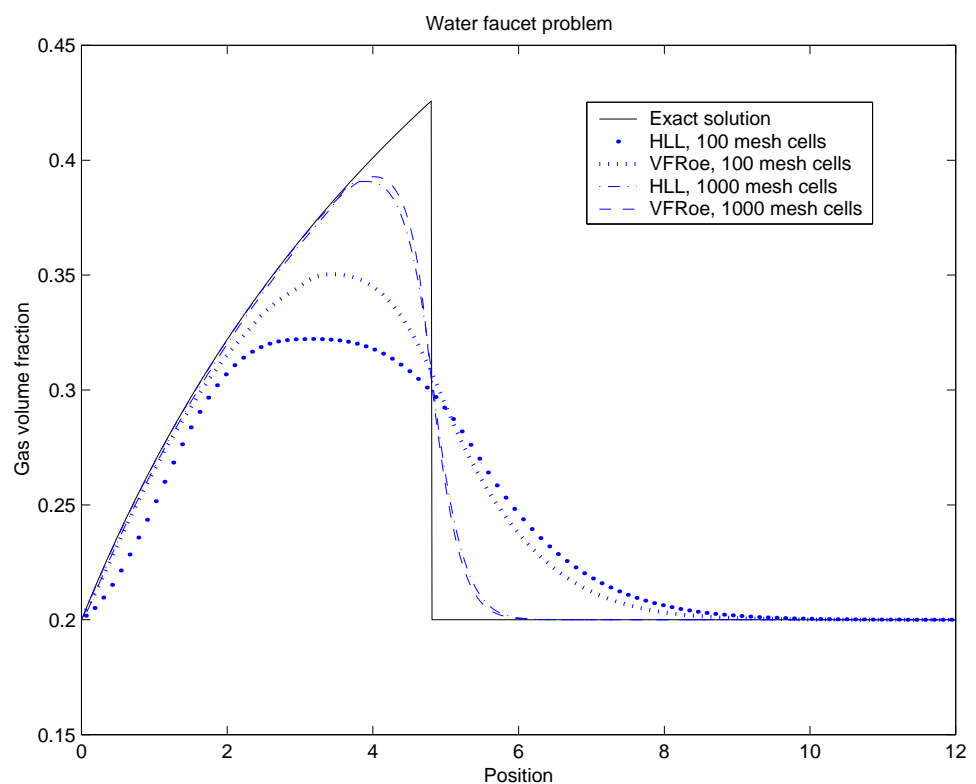

Figure 7: Water faucet problem: gas volume fraction at $t=0.4$.

Under the assumption that the liquid is incompressible and the pressure variation in gas is zero, one can get the exact solution for the evolution of the gas volume fraction,

$$
\alpha_{g}(x, t)= \begin{cases}1-\frac{\left(1-\alpha_{g}^{0}\right) u_{l}^{0}}{\sqrt{2 g x+\left(u_{l}^{0}\right)^{2}}}, & x \leq u_{l}^{0} t+\frac{g t^{2}}{2}, \\ 0.2, & \text { otherwise. }\end{cases}
$$

where $\alpha_{g}^{0}$ is the initial gas volume fraction, $u_{l}^{0}$ the initial liquid velocity, $g$ the gravity acceleration, see e.g. Coquel et. al. [3]. For the numerical solution of the problem, the parameters of the stiffened gas EOS were taken as follows,

$$
\begin{array}{ll}
\text { Liquid } & \text { Gas } \\
\gamma_{l}=4.4 & \gamma_{g}=1.4 \\
\pi_{l}=6 \cdot 10^{6} & \pi_{g}=0 .
\end{array}
$$

For this problem, the velocity relaxation was not used, because the phases have two distinct velocities. The comparison of the numerical results with the exact solution is presented in Fig. 17.

Again, the VFRoe solver gives better results compared to that of HLL solver. The resolution of the discontinuity in gas volume fraction is not perfect, which is due to the following reasons. The sound speed in the liquid is much higher than that of the gas, so 
one has to choose very small time steps in order to satisfy the CFL condition, which leads to numerical inaccuracies. Secondly, the gas pressure is not always constant along the tube, which causes a gas flow in the negative direction and thus smearing of the interface.

\subsection{Mixture Hugoniot test problem}

\subsubsection{Description}

Consider a two-phase mixture, where each component $k$ is governed by the stiffened gas EOS,

$$
P_{k}=\left(\gamma_{k}-1\right) \rho_{k} e_{k}-\gamma_{k} \pi_{k}, \quad k=1,2 .
$$

In this test, we are interested in mixtures of solid materials, which can be considered as compressible under high pressures. One can determine the constants $\gamma_{k}, \pi_{k}$ for some materials from Marsh [11. The corresponding values are summarized in Table 1.

\begin{tabular}{lcc}
\hline & $\gamma$ & $\pi, 10^{9} \mathrm{~Pa}$ \\
\hline Copper & 4.22 & 32.32 \\
Zinc & 4.17 & 15.71 \\
Epoxy & 2.94 & 3.21 \\
Spinel & 1.62 & 141.45 \\
\hline
\end{tabular}

Table 1. Thermodynamic constants for selected materials.

Consider a shock wave propagating in two-phase mixtures of copper/zinc (brass) and epoxy/spinel. Using the constants from Table 1, we can calculate the shock speed in the mixtures of solids with the two-phase flow model and the numerical method described previously. This shock speed can also be estimated from the Rankine-Hugoniot conditions of the mixture Euler equations closed by an appropriate equation of state. Such type of mixture equation of state is described in Massoni et al. [12]. Both numerical results are compared with the experimental data of Marsh [11].

\subsubsection{Two-phase-flow model}

There are no classical Rankine-Hugoniot conditions for the system (2), so we cannot find the shock speed analytically. The approach we use here is straightforward. We calculate the shock speed as the ratio of the biggest (and the only one, in case of a single shock wave) pressure gradient displacement over the time interval.

\subsubsection{Euler equations coupled with the mixture EOS}

For the Euler equations, we can find the shock speed analytically from the Rankine-Hugoniot jump relations. To close the system, we use the mixture EOS due to Massoni et al. [12. It 
is based on the conservation of the energy and mass of the mixture, and on the equality of pressures between phases. It reads,

$$
P=(\Gamma-1) \rho e-\Gamma \Pi,
$$

where $\rho$ is the mixture density, $e$ the mixture internal energy,

$$
\Gamma=1+\frac{1}{\frac{\alpha_{1}}{\gamma_{1}-1}+\frac{\alpha_{2}}{\gamma_{2}-1}}, \quad \Pi=\frac{\Gamma-1}{\Gamma}\left(\alpha_{1} \frac{\gamma_{1} \pi_{1}}{\gamma_{1}-1}+\alpha_{2} \frac{\gamma_{2} \pi_{2}}{\gamma_{2}-1}\right),
$$

and $\alpha_{k}, \gamma_{k}, \pi_{k}$ are the volume fractions and the thermodynamic constants for the phase $k=1,2$. The constants $\Gamma, \Pi$ for the mixtures copper/zinc and epoxy/spinel are given in Table 2.

\begin{tabular}{lcc}
\hline & $\Gamma$ & $\Pi, 10^{9} \mathrm{~Pa}$ \\
\hline Brass (copper/zinc) & 4.20 & 27.49 \\
Epoxy/Spinel & 2.04 & 77.85 \\
\hline
\end{tabular}

Table 2. Thermodynamic constants for selected mixtures.

\subsubsection{Numerical results}

Consider the two sets of initial data for the copper/zinc mixture (brass),

$$
\begin{array}{ll}
\text { Copper } & \text { Zinc } \\
\rho_{1}=8924 \mathrm{~kg} / \mathrm{m}^{3} & \rho_{2}=7139 \mathrm{~kg} / \mathrm{m}^{3} \\
P_{1}=10^{5} \mathrm{~Pa} & P_{2}=10^{5} \mathrm{~Pa} \\
u_{1}=0 \mathrm{~m} / \mathrm{s} & u_{2}=0 \mathrm{~m} / \mathrm{s} \\
\alpha_{1}=0.71 & \alpha_{2}=1-\alpha_{1},
\end{array}
$$

and the epoxy/spinel mixture,

$$
\begin{array}{ll}
\text { Epoxy } & \text { Spinel } \\
\rho_{1}=1185 \mathrm{~kg} / \mathrm{m}^{3} & \rho_{2}=3622 \mathrm{~kg} / \mathrm{m}^{3} \\
P_{1}=10^{5} \mathrm{~Pa} & P_{2}=10^{5} \mathrm{~Pa} \\
u_{1}=0 \mathrm{~m} / \mathrm{s} & u_{2}=0 \mathrm{~m} / \mathrm{s} \\
\alpha_{1}=0.595 & \alpha_{2}=1-\alpha_{1} .
\end{array}
$$

We use a piston boundary condition on the left side to initiate the shock wave. The comparison of the calculated shock speed $U_{s}$ as a function of piston velocity $U_{p}$ with the experimental data of Marsh [11] is presented in Fig. 8

The two-phase flow model gives a very good prediction of the shock speed even on 100 mesh cells compared to the Euler equations for the mixture. Note that the two-phase model 

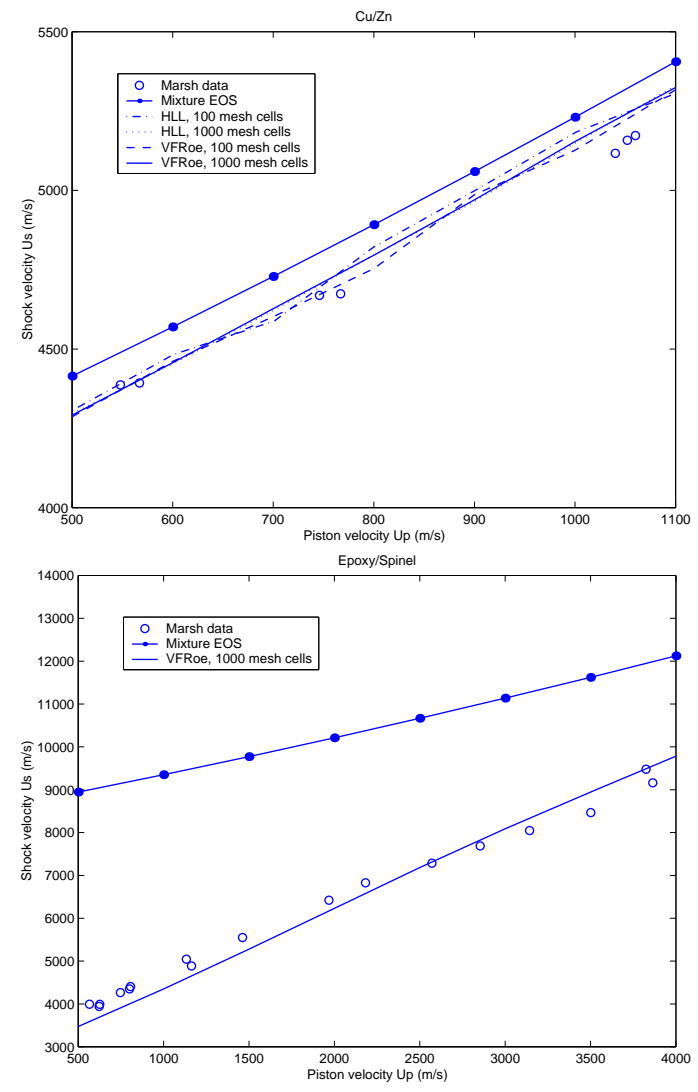

Figure 8: Mixture Hugoniot problem.

does not need any empirically determined parameter. Only the pure material equations of state are used, in conjunction with the hyperbolic solver and relaxation procedures. The results of the VFRoe and HLL solvers do not differ qualitatively on 100 mesh cells. They both show some slight deviations from the experimental results. This is due to the nonaccurate way of determining the shock speed for the two-phase flow model. Nevertheless, the results with 1000 cells show an excellent agreement with the experimental data.

$\mathrm{RR} \mathrm{n}^{\circ} 4247$ 
Andrianov, Saurel \& Warnecke

\section{Conclusions}

In this study, we propose a simple method for compressible two-phase flows. The diffusive Riemann solver used in [15] has been replaced with a more accurate one. The corresponding discretizations of the non-conservative terms have been developed in the absence of pressure or velocity jumps at the volume fraction discontinuity. The comparison of numerical results shows better resolution of the flow discontinuities, obtained by the new scheme. The model and method are validated over several test problems with exact or experimental solutions.

Acknowledgements. The first author gratefully acknowledges the possibility of participating in the summer school CEMRACS'2000 in Marseille and thanks the European Science Foundation (ESF) for the financial support of his stay at the IUSTI, Marseille. He was also partially funded by the DFG-Graduiertenkolleg "Modellierung, Berechnung und Identifikation mechanischer Systeme", Magdeburg, Germany.

\section{References}

[1] R. Abgrall, How to prevent pressure oscillations in multicomponent flow calculations: a quasi conservative approach, J. Comput. Phys. 125, 150-160 (1996).

[2] M.R. Baer, and J. W. Nunziato, A two-phase mixture theory for the deflagration-todetonation transition (DDT) in reactive granular materials, Int. J. of Multiphase Flows, 12, 861-889 (1986).

[3] F. Coquel, K. El Amine, E. Godlewski, B. Perthame, and P. Rascle, A numerical method using upwind schemes for the resolution of two-phase flows, J. Comput. Phys. 136, 272-288 (1997).

[4] R. Fedkiw, B. Merriman, and S. Osher, Simplified discretization of systems of hyperbolic conservation laws containing advection equations, J. Comput. Phys. 157, 302-326 (2000).

[5] T. Gallouet and J.-M. Masella, Un schéma de Godunov approché, C. R. Acad. Sci. Paris Sér. I 323, 77-84 (1996).

[6] S. Gavrilyuk and R. Saurel, A compressible multiphase flow model with microinertia, to appear in J. Comput. Phys. (2001).

[7] K. Gonthier and J. Powers, A high-resolution numerical method for a two-phase model of deflagration-to detonation transition, J. Comput. Phys. 163, 376-433 (2000).

[8] A. Harten and J. M. Hyman, Self-adjusting grid methods for one-dimensional hyperbolic conservation laws, J. Comput. Phys. 50, 235-269 (1983). 
[9] M.-H. Lallemand and R. Saurel, Pressure relaxation procedures for multiphase compressible flows, INRIA Rapport de recherche $n^{0}$ 4038, available online at http://www .inria.fr/rrrt/rr-4038.html.

[10] R. J. LeVeque, Numerical methods for conservation laws, Birkhäuser Verlag, Basel, 1992.

[11] S. P. Marsh, LASL shock Hugoniot data, Univ. of California Press, Berkeley, 1980.

[12] J. Massoni, R. Saurel, B. Nkonga and R. Abgrall, Proposition de méthodes et modèles Eulériens pour les problèmes à interfaces entre fluides compressibles en présence de transfert de chaleur, to appear in Int. J. Heat and Mass Transf. (2001)

[13] V. H. Ransom, Numerical benchmark tests, in Multiphase Science and Technology, vol. 3, G. F. Hewitt, J. M. Delhaye, N. Zuber (eds). Hemisphere, Washington, 1987.

[14] R. Saurel and O. LeMetayer, A multiphase model for compressible flows with interfaces, shocks, detonation waves and cavitation, J. Fluid Mech. 431, 239-271 (2001).

[15] R. Saurel and R. Abgrall, A multiphase Godunov method for compressible multifluid and multiphase flows, J. Comput. Phys. 150, 425-467 (1999).

[16] L. Schwartz, Théorie des distributions, Hermann, Paris, 1966.

\section{Contents}

1 Introduction 3

2 Presentation of the model 5

\begin{tabular}{|lll|}
\hline 3 & Why are the non-conservative terms important & 8
\end{tabular}

$\begin{array}{lll}4 & \text { Numerical method } & 11\end{array}$

4.1 Hvperbolic operaton . . . . . . . . . . . . . . . . . . . . . . . . . 11

$\begin{array}{lll}5 \text { Approximate solution to the Riemann problem } & \mathbf{1 4}\end{array}$

5.1 Conservative svstems . . . . . . . . . . . . . . . . . 14

5.2 Non-conservative svstems . . . . . . . . . . . . . . . . . . 15

$\begin{array}{lll}6 & \text { Extension to the second order } & 17\end{array}$ 
\begin{tabular}{lll}
\hline 7 & Numerical examples & 18
\end{tabular}

7.1 Water-air shock tube . . . . . . . . . . . . . . . . . . . 18

7.2 Water faucet problem . . . . . . . . . . . . . . . . . 20

7.3 Mixture Hugoniot test problem . . . . . . . . . . . . . . . . . . . . . 21

7.3.1 Description . . . . . . . . . . . . . . . . . . 21

7.3 .2 Two-phase-flow model . . . . . . . . . . . . . . . . . . 22

7.3.3 Euler equations coupled with the mixture EOS . . . . . . . . . . 22

7.3 .4 Numerical results . . . . . . . . . . . . . . . . . . . . 22

8 Conclusions 24 


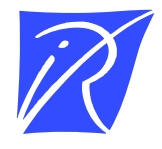

Unité de recherche INRIA Sophia Antipolis 2004, route des Lucioles - BP 93 - 06902 Sophia Antipolis Cedex (France)

Unité de recherche INRIA Lorraine : LORIA, Technopôle de Nancy-Brabois - Campus scientifique 615, rue du Jardin Botanique - BP 101 - 54602 Villers-lès-Nancy Cedex (France)

Unité de recherche INRIA Rennes : IRISA, Campus universitaire de Beaulieu - 35042 Rennes Cedex (France)

Unité de recherche INRIA Rhône-Alpes : 655, avenue de l'Europe - 38330 Montbonnot-St-Martin (France)

Unité de recherche INRIA Rocquencourt : Domaine de Voluceau - Rocquencourt - BP 105 - 78153 Le Chesnay Cedex (France) 